\title{
SYNTHESIS OF NOVEL YLIDENHYDRAZIDES OF 3-BENZYL-8-METHYLXANTHINYL-7-ACETIC ACID AS POTENTIAL BIOLOGICAL ACTIVE COMPOUNDS
}

\author{
Katherine Aleksandrova, Sergii Levich \\ Department of Biological Chemistry, Zaporozhye State Medical University, Zaporozhye, \\ Ukraine
}

\begin{abstract}
In this work, we have described the method of 3-benzyl-8-methylxanthinyl-7-acetohydrazide synthesis and studied its reaction with different aldehydes and ketones in acidic medium. As result, we obtained a series of novel ylidenhydrazides of 3-benzyl-8-methylxanthinyl-7-acetic acid. The structure of the synthesized substances was proved by IR and NMR spectra and elemental analysis and their individuality was confirmed by thin-layer chromatography.
\end{abstract}

Keywords: synthesis, xanthine derivatives, NMR-spectroscopy

\section{INTRODUCTION}

Some of the most widespread drugs are those, which contain in their structures a heterocyclic fragment (1-3). This could be explained by the fact that heterocycle containing compounds play an important role in the metabolic processes. Thus, NAD and NADP (coenzymes of dehydrogenases) are pyridine derivatives, vitamin $\mathrm{B}_{6}$, which is rather important for amino acid metabolism is a pyrimidine derivative, and purine and xanthine heterocycles are part of the nucleotides $(4,5)$.

So chemical modification of well-known natural substances is one of the most promising ways for

\footnotetext{
Address for correspondence:

Sergii Levich

Zaporozhye State University

26 Mayakov'skoho Ave

Zaporizhzhia

Ukraine

e-mail:rshlevas@gmail.com
}

Received: January 5, 2017

Accepted: June 13, 2017 synthesis of novel less toxic biological active compounds, which are also potential medicines.

Xanthine derivatives are handy objects for pharmaceutical research. They are low-toxicity natural compounds with a wide spectrum of pronounced pharmacological properties (antioxidant, diuretic, antibacterial, anti-inflammatory, etc.) and high variability of chemical modification (6-8). At the same time, the hydrazine group is one of the most chemically active functional groups, which easily participates in reactions of nucleophilic addition $(9,10)$ and insertion of such functional fragment to the structure of xanthine molecule could have some positive effect on its synthetic potential (9-11).

In this article we have described the method of synthesis of hydrazide of 3-beznyl-8-methylxanthinyl-7-acetic acid and its $\mathrm{N}$-substituted derivatives and have studied their physicochemical properties.

\section{MATERIALS AND METHODS}

Melting points were determined using the capillary method on DMP (M). ${ }^{1} \mathrm{H}$ NMR-spectra were recorded by Varian Mercury VX-200 device (company «Varian» - USA), solvent - (DMSO-d6), in- 
Synthesis of Novel Ylidenhydrazides of 3-Benzyl-8-Methylxanthinyl-7-Acetic Acid as Potential Biological Active Compounds

ternal standard - TMS. Chemical shifts are reported in ppm (parts per million) values. Infrared (IR) spectra were measured on a Bruker Alpha instrument using a potassium bromide (KBr) disk, scanning from 400 to $4000 \mathrm{~cm}^{-1}$. Elemental analysis of obtained compounds was produced on device Elementar Vario L cube. Analytical thin-layer chromatography (TLC) was carried out on precoated plates, and spots were visualized with ultraviolet (UV) light. Systems, that were used for chromatography: «acetone-propanol-2» 5:2 ratio. All chemicals or reagents were purchased from standard commercial suppliers and treated with standard methods before use.

Propyl 3-benzyl-8-methylxanthinyl-7-acetate 3 was synthesized by a method that we have described earlier (12).

\section{Hydrazide of 3-benzyl-8-methylxanthinyl-7-ace-} tic acid 4

To heated suspension of $0.01 \mathrm{mmol}$ ester 3 in $30 \mathrm{ml}$ of propanol, $5 \mathrm{ml}$ of hydrazine hydrate were added. The solution that formed was refluxed for $30 \mathrm{~min}$. After cooling, a white solid was precipitated. It was filtered out, washed by water and dried at 80-85 ${ }^{\circ} \mathrm{C}$.

Yield 89.1 \%. M.p. $>300^{\circ} \mathrm{C} . \mathrm{R}_{\mathrm{f}}=0.82 . \mathrm{C}_{15} \mathrm{H}_{16} \mathrm{~N}_{6} \mathrm{O}_{3}$. Found, \%: C, 55.17; H, 4.61; N, 25.30. Calculated, \%: C, 54.87; H, 4.91; N, 25.60. IR-spectrum $\left(v, \mathrm{sm}^{-1}\right): 3298$ $(\mathrm{NH}), 3140(\mathrm{NH}), 3002\left(\mathrm{CH}_{\text {arom }}\right), 1710(\mathrm{C}=\mathrm{O}), 1675$ $(\mathrm{C}=\mathrm{O}), 1640(\mathrm{C}=\mathrm{N}), 1592(\mathrm{C}=\mathrm{C}) .{ }^{1} \mathrm{H}$ NMR-spectrum $\left(\delta\right.$-scale, ppm., solvent DMSO- $\left.d_{6}\right): 11.12\left(1 \mathrm{H}, \mathrm{s}, \mathrm{N}^{1} \mathrm{H}\right)$, 9.36 (1H, s, NH), 7.46-7.12 (5H, m, $\left.\mathrm{CH}_{\text {arom }}\right), 5.12(2 \mathrm{H}$, s, $\left.\mathrm{N}^{3}-\mathrm{CH}_{2}\right), 4.98\left(2 \mathrm{H}, \mathrm{s}, \mathrm{N}^{7}-\mathrm{CH}_{2}\right), 4.52\left(2 \mathrm{H}, \mathrm{ws}, \mathrm{NH}_{2}\right)$, $2.35\left(3 \mathrm{H}, \mathrm{s}, \mathrm{C}^{8}-\mathrm{CH}_{3}\right)$.

General procedure for benzylidenhydrazides of -benzyl-8-methylxanthinyl-7-acetic acid synthesis $5 a-1$

Method A. To the solution of $3.28 \mathrm{~g}$ (0.01 mole) hydrazide 4 in $15 \mathrm{ml} 50 \%$ acetic acid, 0.011 mole of aldehyde was added. The mixture was refluxed for 15$30 \mathrm{~min}$. After cooling, a solid was precipitated. It was filtered out, washed by water and dried at $80-85^{\circ} \mathrm{C}$.

Method B. To the solution (heated up to $50{ }^{\circ} \mathrm{C}$ ) of $3.28 \mathrm{~g}$ (0.01 mole) hydrazide 4 in $70 \mathrm{ml}$ aqueous dioxane (1:1), $3 \mathrm{ml}$ of glacial acetic acid and 0.011 mole of aldehyde were added. The mixture was refluxed for 15-20 min. After cooling, a solid was precipitat- ed. The solid was filtered out, washed by water and dried at $80-85^{\circ} \mathrm{C}$.

Ylidenhydrazides $\mathbf{5 p}$-s were synthesized by the same procedure. Ylidenhydrazides 5 m-o were obtained by method B, but the mixture of reagents was not refluxed. It was heated at $80^{\circ} \mathrm{C}$ for $30 \mathrm{~min}$.

Benzylidenhydrazide of -benzyl-8-methylxanthinyl-7-acetic acid (5a)

Yield 96.7 \%. M.p. $>300 \quad{ }^{\circ} \mathrm{C} . \quad \mathrm{R}_{\mathrm{f}}=0.96$. $\mathrm{C}_{22} \mathrm{H}_{20} \mathrm{~N}_{6} \mathrm{O}_{3}$. Found, \%: C, 63.15; H, 5.14; N, 20.48. Calculated, \%: C, 63.45; H, 4.84; N, 20.18. IR-spec$\operatorname{trum}\left(v, \mathrm{sm}^{-1}\right): 3290(\mathrm{NH}), 3120(\mathrm{NH}), 3040\left(\mathrm{CH}_{\text {arom }}\right)$, $1710(\mathrm{C}=\mathrm{O}), 1680(\mathrm{C}=\mathrm{O}), 1640(\mathrm{C}=\mathrm{N}), 1592(\mathrm{C}=\mathrm{C})$. ${ }^{1} \mathrm{H}$ NMR-spectrum $(\delta$-scale, ppm., solvent DMSO$\left.d_{6}\right): 11.78(1 \mathrm{H}, \mathrm{s}, \mathrm{CONH}), 11.14\left(1 \mathrm{H}, \mathrm{s}, \mathrm{N}^{1} \mathrm{H}\right), 8.05(1 \mathrm{H}$, $\mathrm{s}, \mathrm{N}=\mathrm{CH}), 7.74-7.56\left(2 \mathrm{H}, \mathrm{t}, \mathrm{CH}_{\text {arom }}\right), 7.51-7.16(8 \mathrm{H}, \mathrm{m}$, $\left.\mathrm{CH}_{\text {arom }}\right), 5.48\left(2 \mathrm{H}, \mathrm{s}, \mathrm{N}^{7}-\mathrm{CH}_{2}\right), 5.06\left(2 \mathrm{H}, \mathrm{s}, \mathrm{N}^{3}-\mathrm{CH}_{2}\right)$, $2.31\left(3 \mathrm{H}, \mathrm{s}, \mathrm{C}^{8}-\mathrm{CH}_{3}\right)$.

4'-Methylbenzylidenhydrazide of -benzyl8-methylxanthinyl-7-acetic acid (5b)

Yield 81.6 \%. M.p. $289-291{ }^{\circ} \mathrm{C} . \mathrm{R}_{\mathrm{f}}=0.80$. $\mathrm{C}_{23} \mathrm{H}_{22} \mathrm{~N}_{6} \mathrm{O}_{3}$. Found, \%: C, 63.87; H, 5.45; N, 19.82. Calculated, \%: C, 64.17; H, 5.15; N, 19.52. IR-spec$\operatorname{trum}\left(v, \mathrm{sm}^{-1}\right): 3300(\mathrm{NH}), 3120(\mathrm{NH}), 3010\left(\mathrm{CH}_{\text {arom }}\right)$, $1722(\mathrm{C}=\mathrm{O}), 1681(\mathrm{C}=\mathrm{O}), 1620(\mathrm{C}=\mathrm{N}), 1597(\mathrm{C}=\mathrm{C})$. ${ }^{1} \mathrm{H}$ NMR-spectrum $(\delta$-scale, ppm., solvent DMSO$\left.d_{6}\right): 11.72(1 \mathrm{H}, \mathrm{s}, \mathrm{CONH}), 11.17\left(1 \mathrm{H}, \mathrm{s}, \mathrm{N}^{1} \mathrm{H}\right), 7.96(1 \mathrm{H}$, s, $\mathrm{N}=\mathrm{CH}), 7.62-7.51\left(2 \mathrm{H}, \mathrm{t}, \mathrm{CH}_{\text {arom }}\right), 7.34-7.12(7 \mathrm{H}, \mathrm{m}$, $\left.\mathrm{CH}_{\text {arom }}\right), 5.46\left(2 \mathrm{H}, \mathrm{s}, \mathrm{N}^{7}-\mathrm{CH}_{2}\right), 5.09\left(2 \mathrm{H}, \mathrm{s}, \mathrm{N}^{3}-\mathrm{CH}_{2}\right)$, $2.28\left(6 \mathrm{H}, \mathrm{s}, \mathrm{CH}_{3}\right)$.

4'-Chlorobenzylidenhydrazide of -benzyl8-methylxanthinyl-7-acetic acid (5c)

Yield 85.2\%. M.p. $>300{ }^{\circ} \mathrm{C} . \mathrm{R}_{\mathrm{f}}=0.88 . \mathrm{C}_{22} \mathrm{H}_{19} \mathrm{Cl}-$ $\mathrm{N}_{6} \mathrm{O}_{3}$. Found, \%: C, 58.30; H, 4.55; N, 18.34. Calculated, \%: C, 58.60; H, 4.25; N, 18.64. IR-spectrum ( $v$, sm $\left.{ }^{1}\right): 3280(\mathrm{NH}), 3120(\mathrm{NH}), 3040\left(\mathrm{CH}_{\text {arom }}\right), 1720(\mathrm{C}=\mathrm{O})$, $1681(\mathrm{C}=\mathrm{O}), 1650(\mathrm{C}=\mathrm{N}), 1570(\mathrm{C}=\mathrm{C}) .{ }^{1} \mathrm{H}$ NMR-spectrum $\left(\delta\right.$-scale, ppm., solvent DMSO- $\left.d_{6}\right): 11.82(1 \mathrm{H}, \mathrm{s}$, CONH), $11.12\left(1 \mathrm{H}, \mathrm{s}, \mathrm{N}^{1} \mathrm{H}\right), 8.69(1 \mathrm{H}, \mathrm{s}, \mathrm{N}=\mathrm{CH}), 7.89$ $7.81\left(1 \mathrm{H}, \mathrm{d}, \mathrm{CH}_{\text {arom }}\right), 7.77-7.61\left(1 \mathrm{H}, \mathrm{t}, \mathrm{CH}_{\text {arom }}\right), 7.54-$ $7.38\left(2 \mathrm{H}, \mathrm{m}, \mathrm{CH}_{\text {arom }}\right), 7.32-7.17\left(5 \mathrm{H}, \mathrm{m}, \mathrm{CH}_{\text {arom }}\right), 5.47$ $\left(2 \mathrm{H}, \mathrm{s}, \mathrm{N}^{7}-\mathrm{CH}_{2}\right), 5.04\left(2 \mathrm{H}, \mathrm{s}, \mathrm{N}^{3}-\mathrm{CH}_{2}\right), 2.33(3 \mathrm{H}, \mathrm{s}$, $\mathrm{C}^{8}-\mathrm{CH}_{3}$ ).

4'-Brombenzylidenhydrazide of -benzyl-8-methylxanthinyl-7-acetic acid (5d)

Yield $84.3 \%$. M.p. $>300{ }^{\circ} \mathrm{C} . \mathrm{R}_{\mathrm{f}}=0.92 . \mathrm{C}_{22} \mathrm{H}_{19} \mathrm{Br}-$ $\mathrm{N}_{6} \mathrm{O}_{3}$. Found, \%: C, 53.05; H, 4.17; N, 16.67. Calculat- 
Katherine Aleksandrova, Sergii Levich

ed, \%: C, 53.35; H, 3.87; N, 16.63. IR-spectrum ( $v, \mathrm{sm}^{-}$ 1): $3250(\mathrm{NH}), 3160(\mathrm{NH}), 3061\left(\mathrm{CH}_{\text {arom }}\right), 1710(\mathrm{C}=\mathrm{O})$, $1680(\mathrm{C}=\mathrm{O}), 1630(\mathrm{C}=\mathrm{N}), 1570(\mathrm{C}=\mathrm{C}) .{ }^{1} \mathrm{H}$ NMR-spectrum $\left(\delta\right.$-scale, ppm., solvent DMSO- $\left.d_{6}\right): 11.86(1 \mathrm{H}$, s, CONH), $11.18\left(1 \mathrm{H}, \mathrm{s}, \mathrm{N}^{1} \mathrm{H}\right), 8.62(1 \mathrm{H}, \mathrm{s}, \mathrm{N}=\mathrm{CH})$, 7.82-7.51 (7H, m, $\left.\mathrm{CH}_{\text {arom }}\right)$, 7.32-7.14 (2H, m, $\left.\mathrm{CH}_{\text {arom }}\right)$, $5.49\left(2 \mathrm{H}, \mathrm{s}, \mathrm{N}^{7}-\mathrm{CH}_{2}\right), 4.99\left(2 \mathrm{H}, \mathrm{s}, \mathrm{N}^{3}-\mathrm{CH}_{2}\right), 2.31(3 \mathrm{H}$, $\left.\mathrm{s}, \mathrm{C}^{8}-\mathrm{CH}_{3}\right)$.

4'Fluorobenzylidenhydrazide of -benzyl8-methylxanthinyl-7-acetic acid (5e)

Yield $90.5 \%$. M.p. $>300{ }^{\circ} \mathrm{C} . \mathrm{R}_{\mathrm{f}}=0.86 . \mathrm{C}_{22} \mathrm{H}_{19} \mathrm{~F}-$ $\mathrm{N}_{6} \mathrm{O}_{3}$. Found, \%: C, 61.12; H, 4.11; N, 19.65. Calculated, \%: C, 60.82; H, 4.41; N, 19.35. IR-spectrum ( $v$, sm $\left.^{-1}\right): 3317(\mathrm{NH}), 3139(\mathrm{NH}), 3036\left(\mathrm{CH}_{\text {arom }}\right), 1729$ $(\mathrm{C}=\mathrm{O}), 1683(\mathrm{C}=\mathrm{O}), 1667(\mathrm{C}=\mathrm{N}), 1590(\mathrm{C}=\mathrm{C}) .{ }^{1} \mathrm{H}$ NMR-spectrum $\left(\delta\right.$-scale, ppm., solvent DMSO- $\left.d_{6}\right)$ : $11.79(1 \mathrm{H}, \mathrm{s}, \mathrm{CONH}), 11.15\left(1 \mathrm{H}, \mathrm{s}, \mathrm{N}^{1} \mathrm{H}\right), 7.99(1 \mathrm{H}, \mathrm{s}$, $\mathrm{N}=\mathrm{CH}), 7.84-7.62\left(2 \mathrm{H}, \mathrm{m}, \mathrm{CH}_{\text {arom }}\right)$, 7.39-7.18 (7H, m, $\left.\mathrm{CH}_{\text {arom }}\right), 5.46\left(2 \mathrm{H}, \mathrm{s}, \mathrm{N}^{7}-\mathrm{CH}_{2}\right), 5.03\left(2 \mathrm{H}, \mathrm{s}, \mathrm{N}^{3}-\mathrm{CH}_{2}\right)$, $2.35\left(3 \mathrm{H}, \mathrm{s}, \mathrm{C}^{8}-\mathrm{CH}_{3}\right)$.

3',4'-Difluorobenzylidenhydrazide of -benzyl8-methylxanthinyl-7-acetic acid (5f)

Yield $92.1 \%$. M.p. $>300{ }^{\circ} \mathrm{C} . \mathrm{R}_{\mathrm{f}}=0.82 . \mathrm{C}_{22} \mathrm{H}_{18} \mathrm{~F}-$ ${ }_{2} \mathrm{~N}_{6} \mathrm{O}_{3}$. Found, \%: C, 58.71; H, 4.31; N, 18.28. Calculated, \%: C, 58.41; H, 4.01; N, 18.58. IR-spectrum ( $v$, sm $\left.{ }^{1}\right)$ : $3290(\mathrm{NH}), 3150(\mathrm{NH}), 3030\left(\mathrm{CH}_{\text {arom }}\right), 1717(\mathrm{C}=\mathrm{O})$, $1698(\mathrm{C}=\mathrm{O}), 1669(\mathrm{C}=\mathrm{N}), 1584(\mathrm{C}=\mathrm{C}) .{ }^{1} \mathrm{H}$ NMR-spectrum $\left(\delta\right.$-scale, ppm., solvent DMSO- $\left.d_{6}\right): 11.86(1 \mathrm{H}, \mathrm{s}$, $\mathrm{CONH}), 11.15\left(1 \mathrm{H}, \mathrm{s}, \mathrm{N}^{1} \mathrm{H}\right), 7.97(1 \mathrm{H}, \mathrm{s}, \mathrm{N}=\mathrm{CH}), 7.89$ $7.72\left(1 \mathrm{H}, \mathrm{m}, \mathrm{CH}_{\text {arom }}\right), 7.61-7.39\left(2 \mathrm{H}, \mathrm{m}, \mathrm{CH}_{\text {arom }}\right), 7.34-$ $7.12\left(5 \mathrm{H}, \mathrm{m}, \mathrm{CH}_{\text {arom }}\right), 5.44\left(2 \mathrm{H}, \mathrm{s}, \mathrm{N}^{7}-\mathrm{CH}_{2}\right), 5.06(2 \mathrm{H}$, $\left.\mathrm{s}, \mathrm{N}^{3}-\mathrm{CH}_{2}\right), 2.32\left(3 \mathrm{H}, \mathrm{s}, \mathrm{C}^{8}-\mathrm{CH}_{3}\right)$.

4'-N',N'-Dimethylaminobenzylidenhydrazide of -benzyl-8-methylxanthinyl-7-acetic acid (5g)

Yield 89.7 \%. M.p. $283-285{ }^{\circ} \mathrm{C} . \mathrm{R}_{\mathrm{f}}=0.82$. $\mathrm{C}_{24} \mathrm{H}_{25} \mathrm{~N}_{7} \mathrm{O}_{3}$. Found, \%: C, 63.03; H, 5.78; N, 21.74. Calculated, \%: C, 62.73; H, 5.48; N, 21.55. IR-spec$\operatorname{trum}\left(v, \mathrm{sm}^{-1}\right): 3290(\mathrm{NH}), 3130(\mathrm{NH}), 3047\left(\mathrm{CH}_{\text {arom }}\right)$, $1719(\mathrm{C}=\mathrm{O}), 1700(\mathrm{C}=\mathrm{O}), 1642(\mathrm{C}=\mathrm{N}), 1576(\mathrm{C}=\mathrm{C})$. ${ }^{1} \mathrm{H}$ NMR-spectrum $(\delta$-scale, ppm., solvent DMSO$\left.d_{6}\right): 11.49(1 \mathrm{H}, \mathrm{s}, \mathrm{CONH}), 11.10\left(1 \mathrm{H}, \mathrm{s}, \mathrm{N}^{1} \mathrm{H}\right), 7.85(1 \mathrm{H}$, s, $\mathrm{N}=\mathrm{CH}), 7.54-7.46\left(2 \mathrm{H}, \mathrm{d}, \mathrm{CH}_{\text {arom }}\right), 7.38-7.19(5 \mathrm{H}, \mathrm{m}$, $\left.\mathrm{CH}_{\text {arom }}\right), 6.79-6.61\left(2 \mathrm{H}, \mathrm{d}, \mathrm{CH}_{\text {arom }}\right), 5.45\left(2 \mathrm{H}, \mathrm{s}, \mathrm{N}^{7}-\right.$ $\left.\mathrm{CH}_{2}\right), 5.07\left(2 \mathrm{H}, \mathrm{s}, \mathrm{N}^{3}-\mathrm{CH}_{2}\right), 2.94\left(6 \mathrm{H}, \mathrm{s}, \mathrm{N}-\mathrm{CH}_{3}\right), 2.34$ $\left(3 \mathrm{H}, \mathrm{s}, \mathrm{C}^{8}-\mathrm{CH}_{3}\right)$.

4'-Hydroxybenzylidenhydrazide of -benzyl8-methylxanthinyl-7-acetic acid (5h)
Yield 86.3 \%. M.p. $>300 \quad{ }^{\circ} \mathrm{C} . \quad \mathrm{R}_{\mathrm{f}}=0.90$. $\mathrm{C}_{22} \mathrm{H}_{20} \mathrm{~N}_{6} \mathrm{O}_{4}$. Found, \%: C, 60.80; H, 4.36; N, 19.13. Calculated, \%: C, 61.10; H, 4.66; N, 19.43. IR-spec$\operatorname{trum}\left(v, \mathrm{sm}^{-1}\right): 3300(\mathrm{NH}), 3140(\mathrm{NH}), 3020\left(\mathrm{CH}_{\text {arom }}\right)$, $1725(\mathrm{C}=\mathrm{O}), 1689(\mathrm{C}=\mathrm{O}), 1640(\mathrm{C}=\mathrm{N}), 1590(\mathrm{C}=\mathrm{C})$. ${ }^{1} \mathrm{H}$ NMR-spectrum $(\delta$-scale, ppm., solvent DMSO$\left.d_{6}\right): 11.59(1 \mathrm{H}, \mathrm{s}, \mathrm{CONH}), 11.11\left(1 \mathrm{H}, \mathrm{s}, \mathrm{N}^{1} \mathrm{H}\right), 9.89(1 \mathrm{H}$, s, $\mathrm{OH}), 7.88(1 \mathrm{H}, \mathrm{s}, \mathrm{N}=\mathrm{CH}), 7.54-7.48\left(2 \mathrm{H}, \mathrm{d}, \mathrm{CH}_{\text {arom }}\right)$, 7.34-7.19 (5H, m, $\left.\mathrm{CH}_{\text {arom }}\right), 6.85-6.72\left(2 \mathrm{H}, \mathrm{d}, \mathrm{CH}_{\text {arom }}\right)$, $5.45\left(2 \mathrm{H}, \mathrm{s}, \mathrm{N}^{7}-\mathrm{CH}_{2}\right), 5.05\left(2 \mathrm{H}, \mathrm{s}, \mathrm{N}^{3}-\mathrm{CH}_{2}\right), 2.34(3 \mathrm{H}$, $\left.\mathrm{s}, \mathrm{C}^{8}-\mathrm{CH}_{3}\right)$.

4'-Methoxybenzylidenhydrazide of -benzyl8-methylxanthinyl-7-acetic acid (5i)

Yield 95.5 \%. M.p. $>300{ }^{\circ} \mathrm{C} . \quad \mathrm{R}_{\mathrm{f}}=0.94$. $\mathrm{C}_{23} \mathrm{H}_{22} \mathrm{~N}_{6} \mathrm{O}_{4}$. Found, \%: C, 61.57; H, 5.27; N, 19.12 . Calculated, \%: C, 61.87; H, 4.97; N, 18.82. IR-spec$\operatorname{trum}\left(v, \mathrm{sm}^{-1}\right): 3280(\mathrm{NH}), 3160(\mathrm{NH}), 3030\left(\mathrm{CH}_{\text {arom }}\right)$, $1700(\mathrm{C}=\mathrm{O}), 1679(\mathrm{C}=\mathrm{O}), 1660(\mathrm{C}=\mathrm{N}), 1602(\mathrm{C}=\mathrm{C})$. ${ }^{1} \mathrm{H}$ NMR-spectrum $(\delta$-scale, ppm., solvent DMSO$\left.d_{6}\right): 11.64(1 \mathrm{H}, \mathrm{s}, \mathrm{CONH}), 11.13\left(1 \mathrm{H}, \mathrm{s}, \mathrm{N}^{1} \mathrm{H}\right), 7.95(1 \mathrm{H}$, s, $\mathrm{N}=\mathrm{CH}), 7.68-7.55\left(2 \mathrm{H}, \mathrm{d}, \mathrm{CH}_{\text {arom }}\right), 7.49-7.14(5 \mathrm{H}, \mathrm{m}$, $\left.\mathrm{CH}_{\text {arom }}\right), 7.07-6.86\left(2 \mathrm{H}, \mathrm{d}, \mathrm{CH}_{\text {arom }}\right), 5.46\left(2 \mathrm{H}, \mathrm{s}, \mathrm{N}^{7}-\right.$ $\left.\mathrm{CH}_{2}\right), 5.08\left(2 \mathrm{H}, \mathrm{s}, \mathrm{N}^{3}-\mathrm{CH}_{2}\right), 3.72\left(3 \mathrm{H}, \mathrm{s}, \mathrm{OCH}_{3}\right), 2.29$ $\left(3 \mathrm{H}, \mathrm{s}, \mathrm{C}^{8}-\mathrm{CH}_{3}\right)$.

4'-Nitrobenzylidenhydrazide of -benzyl-8-methylxanthinyl-7-acetic acid (5j)

Yield 91.7\%. M.p. $>300^{\circ} \mathrm{C} . \mathrm{R}_{\mathrm{f}}=0.80 . \mathrm{C}_{22} \mathrm{H}_{19} \mathrm{~N}_{7} \mathrm{O}_{5}$. Found, \%: C, 57.56; H, 3.85; N, 20.95. Calculated, \%: C, 57.26; H, 4.15; N, 21.25. IR-spectrum $\left(v, \mathrm{sm}^{-1}\right): 3260$ $(\mathrm{NH}), 3130(\mathrm{NH}), 2997\left(\mathrm{CH}_{\text {arom }}\right), 1703(\mathrm{C}=\mathrm{O}), 1689$ $(\mathrm{C}=\mathrm{O}), 1642(\mathrm{C}=\mathrm{N}), 1583(\mathrm{C}=\mathrm{C}) .{ }^{1} \mathrm{H}$ NMR-spectrum $\left(\delta\right.$-scale, ppm., solvent DMSO- $\left.d_{6}\right): 12.05(1 \mathrm{H}, \mathrm{s}$, CONH), $11.14\left(1 \mathrm{H}, \mathrm{s}, \mathrm{N}^{1} \mathrm{H}\right), 8.12(1 \mathrm{H}, \mathrm{s}, \mathrm{N}=\mathrm{CH}), 8.39$ $8.16\left(2 \mathrm{H}, \mathrm{d}, \mathrm{CH}_{\text {arom }}\right), 8.08-7.86\left(2 \mathrm{H}, \mathrm{d}, \mathrm{CH}_{\text {arom }}\right), 7.39-$ $7.11\left(5 \mathrm{H}, \mathrm{m}, \mathrm{CH}_{\text {arom }}\right), 5.61\left(2 \mathrm{H}, \mathrm{s}, \mathrm{N}^{7}-\mathrm{CH}_{2}\right), 5.09(2 \mathrm{H}$, $\left.\mathrm{s}, \mathrm{N}^{3}-\mathrm{CH}_{2}\right), 2.31\left(3 \mathrm{H}, \mathrm{s}, \mathrm{C}^{8}-\mathrm{CH}_{3}\right)$.

3'-Nitrobenzylidenhydrazide of -benzyl-8-methylxanthinyl-7-acetic acid ( $5 k$ )

Yield 86.8 \%. M.p. $293-294{ }^{\circ} \mathrm{C} . \mathrm{R}_{\mathrm{f}}=0.92$. $\mathrm{C}_{22} \mathrm{H}_{19} \mathrm{~N}_{7} \mathrm{O}_{5}$. Found, \%: C, 57.56; H, 4.45; N, 21.55 . Calculated, \%: C, 57.26; H, 4.15; N, 21.25. IR-spec$\operatorname{trum}\left(v, \mathrm{sm}^{-1}\right): 3340(\mathrm{NH}), 3120(\mathrm{NH}), 3030\left(\mathrm{CH}_{\text {arom }}\right)$, $1700(\mathrm{C}=\mathrm{O}), 1680(\mathrm{C}=\mathrm{O}), 1650(\mathrm{C}=\mathrm{N}), 1590(\mathrm{C}=\mathrm{C})$. ${ }^{1} \mathrm{H}$ NMR-spectrum $(\delta$-scale, ppm., solvent DMSO$\left.d_{6}\right): 12.01(1 \mathrm{H}, \mathrm{s}, \mathrm{CONH}), 11.19\left(1 \mathrm{H}, \mathrm{s}, \mathrm{N}^{1} \mathrm{H}\right), 8.51(1 \mathrm{H}$, s, $\mathrm{N}=\mathrm{CH}), 8.36-8.08\left(3 \mathrm{H}, \mathrm{m}, \mathrm{CH}_{\text {arom }}\right), 7.75-7.61(1 \mathrm{H}$, 
Synthesis of Novel Ylidenhydrazides of 3-Benzyl-8-Methylxanthinyl-7-Acetic Acid as Potential Biological Active Compounds

$\left.\mathrm{t}, \mathrm{CH}_{\text {arom }}\right), 7.41-7.15\left(5 \mathrm{H}, \mathrm{m}, \mathrm{CH}_{\text {arom }}\right), 5.54\left(2 \mathrm{H}, \mathrm{s}, \mathrm{N}^{7}-\right.$ $\left.\mathrm{CH}_{2}\right), 5.04\left(2 \mathrm{H}, \mathrm{s}, \mathrm{N}^{3}-\mathrm{CH}_{2}\right), 2.34\left(3 \mathrm{H}, \mathrm{s}, \mathrm{C}^{8}-\mathrm{CH}_{3}\right)$.

3'-Methoxy-4'-hydroxybenzylidenhydrazide of -benzyl-8-methylxanthinyl-7-acetic acid (5l)

Yield $80.1 \%$. M.p. 274-276 ${ }^{\circ} \mathrm{C} . \mathrm{R}_{\mathrm{f}}=0.82$. $\mathrm{C}_{22} \mathrm{H}_{22} \mathrm{~N}_{6} \mathrm{O}_{5}$. Found, \%: C, 60.03; H, 4.49; N, 18.47. Calculated, \%: C, 59.73; H, 4.79; N, 18.17. IR-spec$\operatorname{trum}\left(v, \mathrm{sm}^{-1}\right): 3270(\mathrm{NH}), 3167(\mathrm{NH}), 3101\left(\mathrm{CH}_{\text {arom }}\right)$, $1712(\mathrm{C}=\mathrm{O}), 1677(\mathrm{C}=\mathrm{O}), 1650(\mathrm{C}=\mathrm{N}), 1590(\mathrm{C}=\mathrm{C})$. ${ }^{1} \mathrm{H}$ NMR-spectrum $(\delta$-scale, ppm., solvent DMSO$\left.d_{6}\right): 11.59(1 \mathrm{H}, \mathrm{s}, \mathrm{CONH}), 11.15\left(1 \mathrm{H}, \mathrm{s}, \mathrm{N}^{1} \mathrm{H}\right), 9.51(1 \mathrm{H}$, s, OH), 7.89 (1H, s, N=CH), 7.34-7.19 (6H, m, $\mathrm{CH}_{a}$ ), 7.14-7.01 (1H, t, $\left.\mathrm{CH}_{\text {arom }}\right)$, 6.82-6.71 $\left(1 \mathrm{H}, \mathrm{d}, \mathrm{CH}_{\mathrm{a}}\right.$ $\left.{ }_{\text {rom }}\right), 5.46\left(2 \mathrm{H}, \mathrm{s}, \mathrm{N}^{7}-\mathrm{CH}_{2}\right), 5.02\left(2 \mathrm{H}, \mathrm{s}, \mathrm{N}^{3}-\mathrm{CH}_{2}\right), 3.79$ $\left(3 \mathrm{H}, \mathrm{s}, \mathrm{OCH}_{3}\right), 2.39\left(3 \mathrm{H}, \mathrm{s}, \mathrm{C}^{8}-\mathrm{CH}_{3}\right)$.

(3-Benzyl-8-methylxanthinyl-7)-acetic acid (2-chloro-quinolin-3-ylmethylene)-hydrazide (5m)

Yield 89.4 \%. M.p. 290-292 ${ }^{\circ} \mathrm{C} . \mathrm{R}_{\mathrm{f}}=0.84$. $\mathrm{C}_{25} \mathrm{H}_{20} \mathrm{ClN}_{7} \mathrm{O}_{3}$. Found, \%: C, 59.52; H, 4.32; N, 19.23. Calculated, \%: C, 59.82; H, 4.02; N, 19.53. IR-spec$\operatorname{trum}\left(v, \mathrm{sm}^{-1}\right): 3290(\mathrm{NH}), 3150(\mathrm{NH}), 3020\left(\mathrm{CH}_{\text {arom }}\right)$, $1700(\mathrm{C}=\mathrm{O}), 1678(\mathrm{C}=\mathrm{O}), 1640(\mathrm{C}=\mathrm{N}), 1574(\mathrm{C}=\mathrm{C})$. ${ }^{1} \mathrm{H}$ NMR-spectrum $(\delta$-scale, ppm., solvent DMSO$\left.d_{6}\right): 12.08(1 \mathrm{H}, \mathrm{s}, \mathrm{CONH}), 11.15\left(1 \mathrm{H}, \mathrm{s}, \mathrm{N}^{1} \mathrm{H}\right), 8.91(1 \mathrm{H}$, s, $\mathrm{N}=\mathrm{CH}), 8.45\left(1 \mathrm{H}, \mathrm{s}, \mathrm{CH}_{\text {arom }}\right), 8.14-8.06\left(1 \mathrm{H}, \mathrm{d}, \mathrm{CH}_{\mathrm{a}-}\right.$ rom $)$, 8.01-7.74 (2H, m, $\left.\mathrm{CH}_{\text {arom }}\right)$, 7.71-7.54 $\left(1 \mathrm{H}, \mathrm{d}, \mathrm{CH}_{\mathrm{a}-}\right.$ rom $)$, 7.39-7.16 (5H, m, $\left.\mathrm{CH}_{\text {arom }}\right), 5.58\left(2 \mathrm{H}, \mathrm{s}, \mathrm{N}^{7}-\mathrm{CH}_{2}\right)$, $5.02\left(2 \mathrm{H}, \mathrm{s}, \mathrm{N}^{3}-\mathrm{CH}_{2}\right), 2.39\left(3 \mathrm{H}, \mathrm{s}, \mathrm{C}^{8}-\mathrm{CH}_{3}\right)$.

(3-Benzyl-8-methylxanthinyl-7)-acetic acid (5-chloro-1,3-dimethyl-1H-pyrazol-4-ylmethylene)hydrazide (5n)

Yield 79.5 \%. M.p. 277-279 ${ }^{\circ} \mathrm{C} . \mathrm{R}_{\mathrm{f}}=0.76$. $\mathrm{C}_{21} \mathrm{H}_{21} \mathrm{ClN}_{8} \mathrm{O}_{3}$. Found, \%: C, 53.49; H, 4.81; N, 24.20. Calculated, \%: C, 53.79; H, 4.51; N, 23.90. IR-spec$\operatorname{trum}\left(v, \mathrm{sm}^{-1}\right): 3310(\mathrm{NH}), 3150(\mathrm{NH}), 3035\left(\mathrm{CH}_{\text {arom }}\right)$, $1723(\mathrm{C}=\mathrm{O}), 1680(\mathrm{C}=\mathrm{O}), 1655(\mathrm{C}=\mathrm{N}), 1595(\mathrm{C}=\mathrm{C})$. ${ }^{1} \mathrm{H}$ NMR-spectrum $(\delta$-scale, ppm., solvent DMSO$\left.d_{6}\right): 11.67(1 \mathrm{H}, \mathrm{s}, \mathrm{CONH}), 11.14\left(1 \mathrm{H}, \mathrm{s}, \mathrm{N}^{1} \mathrm{H}\right), 7.88(1 \mathrm{H}$, s, $\mathrm{N}=\mathrm{CH}), 7.42-7.10\left(5 \mathrm{H}, \mathrm{m}, \mathrm{CH}_{\text {arom }}\right), 5.41\left(2 \mathrm{H}, \mathrm{s}, \mathrm{N}^{7}-\right.$ $\left.\mathrm{CH}_{2}\right), 5.07\left(2 \mathrm{H}, \mathrm{s}, \mathrm{N}^{3}-\mathrm{CH}_{2}\right), 3.71\left(3 \mathrm{H}, \mathrm{s}, \mathrm{CH}_{3}\right), 2.38$ $\left(6 \mathrm{H}, \mathrm{s}, \mathrm{CH}_{3}\right)$.

(3-Benzyl-8-methylxanthinyl-7)-acetic acid (5-chloro-1-methyl-3-ethyl-1H-pyrazol4-ylmethylene)-hydrazide (5o)

Yield 74.7 \%. M.p. 263-264 ${ }^{\circ} \mathrm{C} . \mathrm{R}_{\mathrm{f}}=0.92$. $\mathrm{C}_{22} \mathrm{H}_{23} \mathrm{ClN}_{8} \mathrm{O}_{3}$. Found, \%: C, 55.05; H, 5.10; N, 23.50. Calculated, \%: C, 54.72; H, 4.80; N, 23.20. IR-spec- $\operatorname{trum}\left(v, \mathrm{sm}^{-1}\right): 3260(\mathrm{NH}), 3110(\mathrm{NH}), 3020\left(\mathrm{CH}_{\text {arom }}\right)$, $1710(\mathrm{C}=\mathrm{O}), 1696(\mathrm{C}=\mathrm{O}), 1633(\mathrm{C}=\mathrm{N}), 1580(\mathrm{C}=\mathrm{C})$. ${ }^{1} \mathrm{H}$ NMR-spectrum $(\delta$-scale, ppm., solvent DMSO$\left.d_{6}\right): 11.69(1 \mathrm{H}, \mathrm{s}, \mathrm{CONH}), 11.11\left(1 \mathrm{H}, \mathrm{s}, \mathrm{N}^{1} \mathrm{H}\right), 7.86(1 \mathrm{H}$, s, $\mathrm{N}=\mathrm{CH}), 7.35-7.16\left(5 \mathrm{H}, \mathrm{m}, \mathrm{CH}_{\text {arom }}\right), 5.43\left(2 \mathrm{H}, \mathrm{s}, \mathrm{N}^{7}-\right.$ $\left.\mathrm{CH}_{2}\right), 5.05\left(2 \mathrm{H}, \mathrm{s}, \mathrm{N}^{3}-\mathrm{CH}_{2}\right), 3.76\left(3 \mathrm{H}, \mathrm{s}, \mathrm{CH}_{3}\right), 2.77$ $\left(2 \mathrm{H}, \mathrm{q}, \mathrm{CH}_{2}\right), 2.32\left(3 \mathrm{H}, \mathrm{s}, \mathrm{C}^{8}-\mathrm{CH}_{3}\right), 1.15\left(3 \mathrm{H}, \mathrm{t}, \mathrm{CH}_{3}\right)$.

(3-Benzyl-8-methylxanthinyl-7)-acetic acid [3-(5-nitro-furan-2-yl)-allylidene]-hydrazide (5p)

Yield $76.3 \%$. M.p. 227-228 ${ }^{\circ} \mathrm{C} . \mathrm{R}_{\mathrm{f}}=0.92$. $\mathrm{C}_{22} \mathrm{H}_{19} \mathrm{~N}_{7} \mathrm{O}_{6}$. Found, \%: C, 55.05; H, 4.31; N, 20.24 . Calculated, \%: C, 55.35; H, 4.01; N, 20.54. IR-spec$\operatorname{trum}\left(v, \mathrm{sm}^{-1}\right): 3260(\mathrm{NH}), 3139(\mathrm{NH}), 3033\left(\mathrm{CH}_{\text {arom }}\right)$, $1700(\mathrm{C}=\mathrm{O}), 1680(\mathrm{C}=\mathrm{O}), 1660(\mathrm{C}=\mathrm{N}), 1560(\mathrm{C}=\mathrm{C})$. ${ }^{1} \mathrm{H}$ NMR-spectrum $(\delta$-scale, ppm., solvent DMSO$\left.d_{6}\right): 11.86(1 \mathrm{H}, \mathrm{s}, \mathrm{CONH}), 11.14\left(1 \mathrm{H}, \mathrm{s}, \mathrm{N}^{1} \mathrm{H}\right), 7.82(1 \mathrm{H}$, s, $\mathrm{N}=\mathrm{CH}), 7.74(1 \mathrm{H}, \mathrm{d}, \mathrm{CH}), 7.41-7.15\left(5 \mathrm{H}, \mathrm{m}, \mathrm{CH}_{\mathrm{a}}\right.$ rom $), 7.12(1 \mathrm{H}, \mathrm{d}, \mathrm{CH}), 7.09-6.96\left(2 \mathrm{H}, \mathrm{d}, \mathrm{CH}_{\text {arom }}\right), 5.39$ $\left(2 \mathrm{H}, \mathrm{s}, \mathrm{N}^{7}-\mathrm{CH}_{2}\right), 5.06\left(2 \mathrm{H}, \mathrm{s}, \mathrm{N}^{3}-\mathrm{CH}_{2}\right), 2.29(3 \mathrm{H}, \mathrm{s}$, $\left.\mathrm{C}^{8}-\mathrm{CH}_{3}\right)$.

(3-Benzyl-8-methylxanthinyl-7)-acetic acid [5-(4-nitro-phenyl)-furan-2-ylmethylene]-hydrazide (5q)

Yield $71.5 \%$. M.p. 209-211 ${ }^{\circ} \mathrm{C} . \mathrm{R}_{\mathrm{f}}=0.78$. $\mathrm{C}_{26} \mathrm{H}_{21} \mathrm{~N}_{7} \mathrm{O}_{6}$. Found, \%: C, 59.50; H, 4.31; N, 18.29. Calculated, \%: C, 59.20; H, 4.01; N, 18.59. IR-spec$\operatorname{trum}\left(v, \mathrm{sm}^{-1}\right): 3260(\mathrm{NH}), 3109(\mathrm{NH}), 3080\left(\mathrm{CH}_{\text {arom }}\right)$, $1713(\mathrm{C}=\mathrm{O}), 1679(\mathrm{C}=\mathrm{O}), 1658(\mathrm{C}=\mathrm{N}), 1597(\mathrm{C}=\mathrm{C})$. ${ }^{1} \mathrm{H}$ NMR-spectrum $(\delta$-scale, ppm., solvent DMSO$\left.d_{6}\right): 11.88(1 \mathrm{H}, \mathrm{s}, \mathrm{CONH}), 11.15\left(1 \mathrm{H}, \mathrm{s}, \mathrm{N}^{1} \mathrm{H}\right), 7.96(1 \mathrm{H}$, s, $\mathrm{N}=\mathrm{CH}), 8.31-8.16\left(2 \mathrm{H}, \mathrm{d}, \mathrm{CH}_{\text {arom }}\right), 7.94-7.82(2 \mathrm{H}, \mathrm{d}$, $\left.\mathrm{CH}_{\text {arom }}\right)$, 7.43-7.37 (1H, d, $\left.\mathrm{CH}_{\text {arom }}\right)$, 7.36-7.18 (5H, m, $\left.\mathrm{CH}_{\text {arom }}\right)$, 7.14-7.06 (1H, d, $\left.\mathrm{CH}_{\text {arom }}\right), 5.51\left(2 \mathrm{H}, \mathrm{s}, \mathrm{N}^{7}-\right.$ $\left.\mathrm{CH}_{2}\right), 5.07\left(2 \mathrm{H}, \mathrm{s}, \mathrm{N}^{3}-\mathrm{CH}_{2}\right), 2.34\left(3 \mathrm{H}, \mathrm{s}, \mathrm{C}^{8}-\mathrm{CH}_{3}\right)$.

3-benzyl-8-methylxanthinyl-7-N'-[2-oxo-2,3-dihydro-1H-indol-3-ylidene]acetohydrazide (5r)

Yield $92.3 \%$. M.p. $>300{ }^{\circ} \mathrm{C} . \quad \mathrm{R}_{\mathrm{f}}=0.86$. $\mathrm{C}_{23} \mathrm{H}_{19} \mathrm{~N}_{7} \mathrm{O}_{4}$. Found, \%: C, 60.09; H, 3.89; N, 21.13. Calculated, \%: C, 60.39; H, 4.19; N, 21.43. IR-spec$\operatorname{trum}\left(v, \mathrm{sm}^{-1}\right): 3300(\mathrm{NH}), 3180(\mathrm{NH}), 3050\left(\mathrm{CH}_{\text {arom }}\right)$, $1720(\mathrm{C}=\mathrm{O}), 1680(\mathrm{C}=\mathrm{O}), 1660(\mathrm{C}=\mathrm{N}), 1590(\mathrm{C}=\mathrm{C})$. ${ }^{1} \mathrm{H}$ NMR-spectrum $(\delta$-scale, ppm., solvent DMSO$\left.d_{6}\right): 12.72(1 \mathrm{H}, \mathrm{s}, \mathrm{NH}), 11.32(1 \mathrm{H}, \mathrm{s}, \mathrm{CONH}), 11.14$ $\left(1 \mathrm{H}, \mathrm{s}, \mathrm{N}^{1} \mathrm{H}\right), 7.61-7.50\left(1 \mathrm{H}, \mathrm{d}, \mathrm{CH}_{\text {arom }}\right), 7.48-7.19(6 \mathrm{H}$, $\left.\mathrm{m}, \mathrm{CH}_{\text {arom }}\right), 7.17-6.92\left(1 \mathrm{H}, \mathrm{t}, \mathrm{CH}_{\text {arom }}\right), 6.91-6.83(1 \mathrm{H}, \mathrm{d}$, $\left.\mathrm{CH}_{\text {arom }}\right), 5.67\left(2 \mathrm{H}, \mathrm{s}, \mathrm{N}^{7}-\mathrm{CH}_{2}\right), 5.09\left(2 \mathrm{H}, \mathrm{s}, \mathrm{N}^{3}-\mathrm{CH}_{2}\right)$, $2.36\left(3 \mathrm{H}, \mathrm{s}, \mathrm{C}^{8}-\mathrm{CH}_{3}\right)$. 
(3-Benzyl-8-methylxanthinyl-7)-acetic acid [1-(4-amino-phenyl)-ethylidene]-hydrazide (5s)

Yield 69.8\%.M.p. $>300^{\circ} \mathrm{C} . \mathrm{R}_{\mathrm{f}}=0.72 . \mathrm{C}_{23} \mathrm{H}_{22} \mathrm{~N}_{6} \mathrm{O}_{3}$. Found, \%: C, 64.47; H, 5.45; N, 19.22. Calculated, \%: C, 64.17; H, 5.15; N, 19.52. IR-spectrum $\left(v, \mathrm{sm}^{-1}\right): 3250$ $(\mathrm{NH}), 3140(\mathrm{NH}), 3020\left(\mathrm{CH}_{\text {arom }}\right), 1710(\mathrm{C}=\mathrm{O}), 1692$ $(\mathrm{C}=\mathrm{O}), 1649(\mathrm{C}=\mathrm{N}), 1587(\mathrm{C}=\mathrm{C}) .{ }^{1} \mathrm{H}$ NMR-spectrum $\left(\delta\right.$-scale, ppm., solvent DMSO- $\left.d_{6}\right): 11.12\left(1 \mathrm{H}, \mathrm{s}, \mathrm{N}^{1} \mathrm{H}\right)$, $10.75(1 \mathrm{H}, \mathrm{s}, \mathrm{CONH}), 7.52-7.46\left(2 \mathrm{H}, \mathrm{d}, \mathrm{CH}_{\text {arom }}\right), 7.41-$ $7.14\left(5 \mathrm{H}, \mathrm{m}, \mathrm{CH}_{\text {arom }}\right), 6.59-6.52\left(2 \mathrm{H}, \mathrm{d}, \mathrm{CH}_{\text {arom }}\right), 6.09$ $\left(2 \mathrm{H}, \mathrm{s}, \mathrm{NH}_{2}\right), 5.51\left(2 \mathrm{H}, \mathrm{s}, \mathrm{N}^{7}-\mathrm{CH}_{2}\right), 5.11\left(2 \mathrm{H}, \mathrm{s}, \mathrm{N}^{3}-\right.$ $\left.\mathrm{CH}_{2}\right), 2.34\left(3 \mathrm{H}, \mathrm{s}, \mathrm{C}^{8}-\mathrm{CH}_{3}\right), 2.12\left(3 \mathrm{H}, \mathrm{s}, \mathrm{CH}_{3}\right)$.

\section{RESULTS AND DISCUSSION}

In the previous works we have described the method of propyl 3-benzyl-8-methylxanthinyl-7-acetate synthesis from 1-benzyl-5.6-diaminouracil (Fig. 1) (12).

In continuation of our search for potential biological active compounds among 3-beznylxanthine derivatives we obtained hydrazide of 3-beznylxan- thinyl-7-acetic acid $\mathbf{4}$ by the reaction of ester $\mathbf{3}$ with hydrazine hydrate (Fig. 2).

At ${ }^{1} \mathrm{H}$ NMR-spectra of hydrazide 4 signals of methyl and methylene groups of ester residue were absent, but signals of hydrazide residue protons at $9.37 \mathrm{ppm}(1 \mathrm{H}, \mathrm{s})$ and $4.52 \mathrm{ppm}(2 \mathrm{H}$, w.s. $)$ were present. These and also intensive singlet of methylene group, that combined with a Nitrogen atom at position 7 , at $4.98 \mathrm{ppm}$ proved the presence of acethydrazide residue in the structure of compound 4.

At the next stage we studied the reaction of hydrazide 4 with aliphatic, aromatic and heterocyclic carbonyl-containing compounds and obtained appropriate ylidenehydrazides 5a-s (Fig. 3). Reaction was conducted by reflux of reagents in $50 \%$ acetic acid or aqueous dioxane in the presence of catalytic amount of glacial acetic acid.

Ylidenehydrazides 5a-s are white, daffodil, yellow or orange crystal compounds, insoluble in wa-<smiles>CCCOC(=O)Cn1c(C)nc2c1c(=O)[nH]c(=O)n2Cc1ccccc1</smiles>

a) acetic acid, reflux; ø) $\mathrm{NaOH}, \mathrm{H}_{2} \mathrm{O}$, reflux; c) propyl ch loroacetate, $\mathrm{NaHCO}_{3}$, DMF, reflux.

Figure 1. Scheme of propyl 3-benzyl-8-methylxanthinyl-7-acetate synthesis

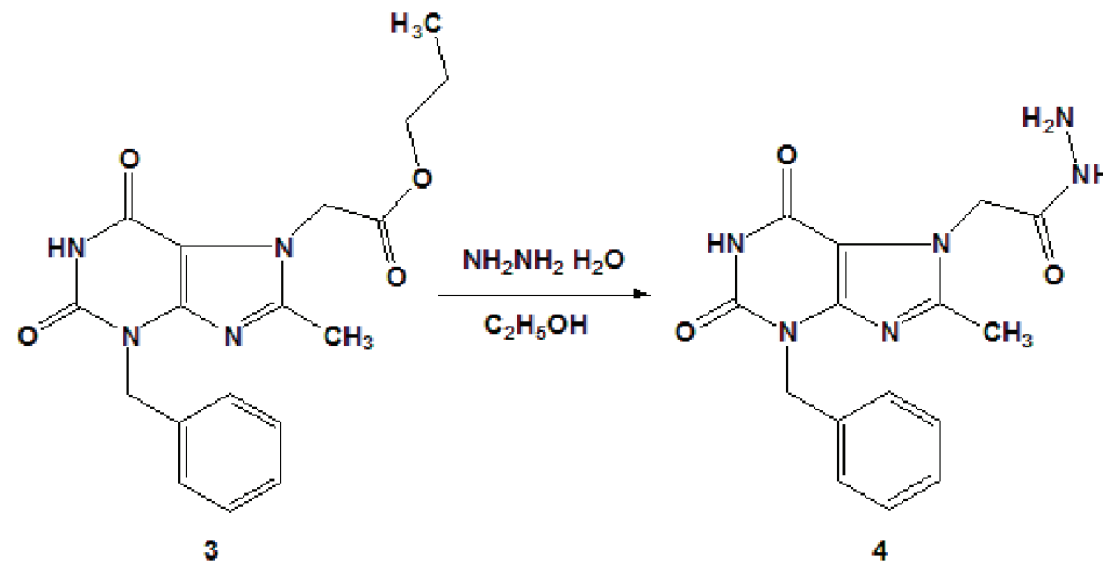

Figure 2. Scheme of hydrazide of 3-benzyl-8-methylxanthinyl-7-acetic acid synthesis 


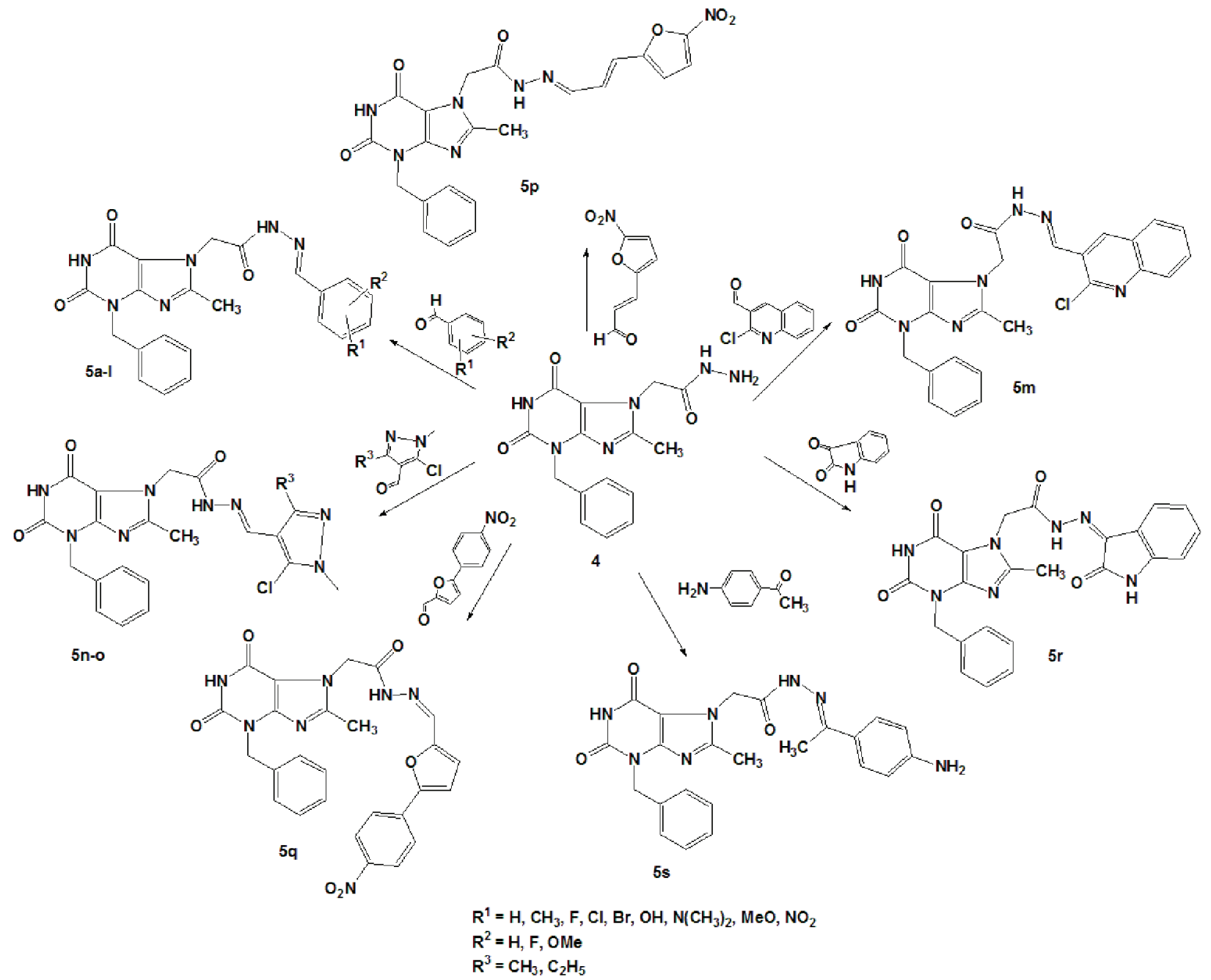

Figure 3. Scheme of ylidenhydrazides of 3-benzyl-8-methylxanthinyl-7-acetic acid synthesis

ter, diethyl ether, soluble in DMSO, ethanol and propanol.

In IR-spectra of compounds 5a-s were present strips of absorption of $\mathrm{N}-\mathrm{H}, \mathrm{C}=\mathrm{O}, \mathrm{C}=\mathrm{C}$ and $\mathrm{C}=\mathrm{N}$.

In ${ }^{1} \mathrm{H}$ NMR-spectra of ylidenehydrazides in comparison with initial hydrazide spectrum was absent signal of $\mathrm{NH}_{2}$-group. At the same time, singlets of methine groups protons (at interval 8.91-7.82 ppm) were registered. Protons of NH-group of hydrazide residue resonated at a more weak field at 12.08-11.49 ppm.

In the spectra of all ylidenehydrazides signals of appropriate ylidene residues protons were also registered.

Thus, presence of 4-hydroxybenzylidene fragment of the ${ }^{1} \mathrm{H}$ NMR-spectra of compound $5 \mathbf{h}$ was proved by singlet of $\mathrm{OH}$-group proton (at $9.89 \mathrm{ppm}$ ) and two doublets of protons of p-substituted benzylidene residue (at 7.54-7.48 ppm and 6.85-6.72 ppm). Substituents at 4 position of benzene ring of compounds $\mathbf{5 b}, \mathbf{5 g}$ and $5 \mathbf{i}$ were proved by singlets of methyl at $2.28 \mathrm{ppm}$ (compound $\mathbf{5 b}$ ) and $2.94 \mathrm{ppm}$ (compound $5 \mathrm{~g}$ ) and methoxy groups at $3.72 \mathrm{ppm}$ (compound 5i).

Synthesis of 3-benzyl-8-methylxanthinyl7-N'-[2-oxo-2,3-dihydro-1H-indol-3-ylidene]acetohydrazide (compound $5 \mathbf{r}$ ) was confirmed by the presence of a signal of $\mathrm{NH}$-group of indole fragments at $12.72 \mathrm{ppm}$ and increasing of the aromatic proton multiplet intensity up to 9 . 


\section{CONCLUSIONS}

The developed method could be used for hydrazides and ylidenhydrazides of 3-aralkylxanthinyl7 -acetic acids synthesis. The PASS program prognosis confirmed that obtained compounds could have anti-bacterial and antifungal activities, which would be researched in further studies.

\section{REFERENCES}

1. Saini MS, Kumar A, Dwivedi J, Singh R. A Review: Biological significances of heterocyclic compounds. Int J Pharm Sci Res. 2013;3(4):66-77.

2. Joule JA, Mills K. Heterocycles in Nature. In: Heterocyclic Chemistry at a Glance. Chichester: John Wiley \& Sons, Ltd.; 2010. p. 158-166.

3. Joule JA, Mills K Heterocycles in Medicine. In: Heterocyclic Chemistry at a Glance. Chichester: John Wiley \& Sons, Ltd.; 2010, p. 167-179.

4. Murray RK, Granner DK, Mayes PA, Rodwell VW. Harper's Illustrated Biochemistry. 26th ed. India: LANGE medical books; 2006.

5. Satyanarayana U, Chakrapani U. Biochemistry. 3rd ed. Kolkata: Books and Allied; 2006.

6. Mohamed T, Osman W, Tin G, Rao PN. Selective inhibition of human acetylcholinesterase by xanthine derivatives: In vitro inhibition and molecular modeling investigations. Bioorg Med Chem Lett. 2013; 23(15):4336-41. doi: 10.1016/j. bmcl.2013.05.092

7. Mak G, Hanania NA. New bronchodilators. Curr Opin Pharmacol. 2012; 12(3): 238-45. doi: 10.1016/j. coph.2012.02.019

8. Song B, Xiao T, Xiaolu Q. Design and synthesis of 8-substituted benzamido-phenylxanthine derivatives as MAO-B inhibitors. Bioorg Med Chem Lett. 2012;22(4):1739-42. doi: 10.1016/j.bmcl.2011.12.094

9. Romanenko NI, Nazarenko, MV, Ivanchenko DG, Pakhomova OO, Sharapova TA. The study of reactions of 7-substituted 8-hydrazino-3-methylxanthine with $\beta$-dicarbonyl compounds. Aktualni pytannia farmatsevtychnoi i medychnoi nauky ta praktyky. 2015;2(18):4-8. doi: 10.14739/2409-2932.2015.2.45125

10. Ivanchenko DG. Synthesis, physical-chemical and biological properties of 1,8-disubstituted of theobromine. V. 8-Benzylidenhydrazino1-p-methylbenzyltheobromines. Zaporozhskij medicinskij zhurnal. 2015;5(92):89-92. doi: 10.14739/2310-1210.2015.5.53768
11. Ivanchenko DH, Romanenko MI, Sharapova TA, Aleksandrova KV, Kamyshny AM, Polishchuk NM. Synthesis and antibacterial properties of 8-beznylidenhydrazine-1-propyltheobromines. Aktualni pytannia farmatsevtychnoi i medychnoi nauky ta praktyky. 2015;1(17):51-5.

12. Levich SV, Shkoda AS, Aleksandrova KV. Synthesis and physicochemical properties of S-substituted derivatives of 3-benzyl-8-methyl-7-[(4-phenyl5-thio-4H-1,2,4-triazolyl-3)-methyl]xanthine. Aktualni pytannia farmatsevtychnoi i medychnoi nauky ta praktyky. 2013;1(11):54-8. 